\title{
Collisional damping of zonal flows due to finite Larmor radius effects
}

\author{
Paolo Ricci, ${ }^{1, a)}$ B. N. Rogers, ${ }^{2, b)}$ and W. Dorland ${ }^{3, c)}$ \\ ${ }^{1}$ Centre de Recherches en Physique des Plasmas, École Polytechnique Fédérale de Lausanne, \\ Association EURATOM-Confédération Suisse, CH-1015 Lausanne, Switzerland \\ ${ }^{2}$ Department of Physics and Astronomy, Dartmouth College, Hanover, New Hampshire 03755, USA \\ ${ }^{3}$ Department of Physics, University of Maryland, College Park, Maryland 20742, USA
}

(Received 27 January 2010; accepted 16 May 2010; published online 12 July 2010)

\begin{abstract}
The collisional damping of seeded $\mathbf{E} \times \mathbf{B}$ zonal flows on the ion Larmor radius scale is studied using a gyrokinetic model. The focus is on flow damping due to finite Larmor radius effects, which cause a $v_{\|} / v$ anisotropy of the ion distribution function that is damped by ion-ion collisions. The gyrokinetic equations are solved in a slab geometry with no gradients or curvature, and a gyroaveraged Lorentz collision operator that conserves particle number, momentum, and energy is used. The solution of the gyrokinetic equations explores the dependence of the damping rate on the wavelength of the flows and the impact of the collisions on the ion distribution function. These numerical results can be used as a benchmark test during the implementation of finite Larmor radius effects in the collision operator of gyrokinetic codes. (C) 2010 American Institute of Physics.
\end{abstract}

[doi:10.1063/1.3447875]

\section{INTRODUCTION}

$\mathbf{E} \times \mathbf{B}$ zonal flows play an important role in many magnetically confined, magnetohydrodynamically (MHD) stable plasma systems. ${ }^{1,2}$ The bulk of heat and particle transport in such systems is typically driven by residual, non-MHD, small-scale modes, which produce turbulent convection within the plasma. The zonal flows in these systems have a stabilizing impact on the turbulence that can become dramatically strong as the system approaches marginal stability: turbulence suppression due to zonal flows has been observed, for example, in ion temperature gradient, ${ }^{3-5}$ electron temperature gradient, ${ }^{6}$ trapped electron, ${ }^{7,8}$ and entropy ${ }^{9,10}$ mode turbulence. In these cases the turbulence-driving modes can be virtually eliminated by zonal flows that are themselves weak enough to be stable to Kelvin-Helmholtz-like instabilities and other modes. ${ }^{11}$ The result is a quasistatic, long-lived zonal-flow dominated state in which the transport is negligibly small.

In this context, collisional effects can significantly decrease the amplitude of the zonal flows (see, e.g., Refs. 12-16) and can ultimately lead to a reduced suppression of the turbulent transport, as investigated through gyrokinetic (see, e.g., Refs. 17-19) and fluid simulations (see, e.g., Ref. 20).

Herein we focus on the collisional damping of zonal flows on the ion Larmor radius scale by studying the initial value problem of the evolution of a seeded zonal flow. To simplify the problem as much as possible, we consider a plasma without magnetic curvature or background gradients. We focus on the cases where the initial gyrocenter distribution functions are isotropic Maxwellians and the seeded zonal flows are associated with finite-Larmour radius (FLR) perturbations of the ion distribution function. The FLR per-

\footnotetext{
${ }^{a}$ Electronic mail: paolo.ricci@epfl.ch.

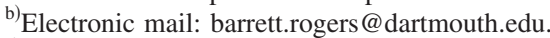

${ }^{c)}$ Electronic mail: bdorland@umd.edu.
}

turbation of the distribution function depends on $v_{\|} / v$, and thus the particle distribution function results to be nonisotropic. Because collisions in the system tend to isotropize the distribution functions, the zonal flows related to this nonisotropy are also damped out by collisions. As we show here, this damping is to some extent dependent on the initial conditions. Moreover, since FLR effects depend on the value of $k \rho_{i}$ of the perturbations, there is also a strong $k$ dependence of the damping. Our study focuses on the damping on the collisional ion time scale. We do not consider the damping on the (much slower) electron time scale.

Collisional damping of zonal flow due to FLR effects has been found particularly important in the $\mathrm{Z}$ pinch system. ${ }^{9}$ In this configuration, $\mathbf{E} \times \mathbf{B}$ zonal flows are stationary solutions of the collisionless gyrokinetic equation and are stable to secondary instabilities, such as the Kelvin-Helmholtz instability, in the weakly driven regimes. These flows can only be damped by collisions that involve particles streaming at different velocities, i.e., with different gyrocenter positions. These collisions are made possible by the particle Larmor motion. In fact, in the $\mathrm{Z}$ pinch, the damping of the zonal flows due to FLR collisional effects was shown to increase the plasma turbulence and particle transport by two orders of magnitude or more $;{ }^{9}$ the analysis of the dipolar configuration also led to very similar findings. ${ }^{10}$

Assuming $\nu_{i} \ll \Omega_{c i}$, we use the gyrokinetic model to describe the collisional zonal flow damping (see Ref. 21 and also Ref. 22 for a recent review on the gyrokinetic model), by carrying out simulations with the GS2 code. ${ }^{23,24}$ The deduction of an accurate and complete collision operator in the context of gyrokinetic simulation is described in Refs. 25 and 26 and its numerical implementation in GS2 has been recently described in Ref. 27. Here, however, since the Lorentz part of the collision operator that describes pitchangle scattering is responsible for the damping of the $v_{\|} / v$ anisotropy, we neglect, for simplicity, the energy diffusion part. As a further check on our results, we also benchmark 
the GS2 results against an independent gyrokinetic code based on an entirely different numerical approach. Thanks to their reliability, the numerical results presented here can be used as a benchmark test during the implementation of FLR effects in the collision operator of gyrokinetic codes.

Our main results may be summarized as follows. First, the damping vanishes at long wavelengths as $\left(k \rho_{i}\right)^{2}$ and it is stronger than the $\left(k \rho_{i}\right)^{2}$ scaling at shorter wavelengths. Second, we show that collisions affect the distribution function mostly at higher energies on the order of $v / v_{\text {th }} \simeq 1.5$. Finally, we point out that a proper account of FLR effects in the collision operator can be essential to correctly describe the damping. In particular, we show that neglecting the momentum conserving terms can lead to unphysical results.

This paper is organized as follows. We describe in Sec. II the collision operator in the gyrokinetic model. In Sec. III we discuss the time scales related to the collision operator and in Sec. IV we present our numerical results, obtained by solving the gyrokinetic equations. The conclusions follow. In the appendixes, we describe the detailed derivation of the collision operator (Appendix A) and the study of the unphysical results that are obtained if simplified versions of the collision operator are used that do not properly take into account ion Larmor radius effects (Appendix B).

\section{THE COLLISION OPERATOR IN THE GYROKINETIC MODEL}

In order to simplify the gyrokinetic model as much as possible and focus on the collisional damping of flows perpendicular to the magnetic field, we consider a homogeneous plasma with no curvature, density, or temperature gradients, $k_{\|}=0$, and we neglect electromagnetic effects. With these assumptions, the linearized gyrokinetic equations for electrons and ions $(\alpha=i, e)$ assume the following form: ${ }^{21,22}$

$$
\frac{\partial h_{\alpha}}{\partial t}=-q_{\alpha} \frac{d \phi}{d t} \frac{\partial F_{0 \alpha}}{\partial \epsilon} J_{0}\left(\frac{k_{\perp} v_{\perp}}{\Omega_{\alpha}}\right)+\mathcal{I}_{\alpha}\left(f_{\alpha}\right)
$$

where the collision operator $\mathcal{I}_{\alpha}$ is related to the collision operator in real space $\mathcal{C}_{\alpha}$ by

$$
\mathcal{I}_{\alpha}\left(f_{\alpha}\right)=\int_{-\pi}^{\pi} \frac{d \zeta}{2 \pi} \exp \left(-i L_{\alpha}\right) \mathcal{C}_{\alpha}\left(f_{\alpha}\right)
$$

and $\phi$ is the electrostatic potential, $\zeta$ is the gyroangle variable, $q_{\alpha}$ and $\Omega_{\alpha}$ are the charge and cyclotron frequency for the species $\alpha, L_{\alpha}=(\mathbf{v} \times \mathbf{b} \cdot \mathbf{k}) / \Omega_{\alpha}$, and $F_{0 \alpha}$ is the equilibrium distribution function (in the remainder of the present paper, we assume that $F_{0 \alpha}$ is an isotropic Maxwellian distribution with temperature $T_{\alpha}$ and density $n_{0}$ ). The nonadiabatic part of the distribution function $h_{\alpha}=h_{\alpha}(\mathbf{k}, \epsilon, \mu)$ (with $\epsilon=m_{\alpha} v^{2} / 2$ and $\left.\mu=m_{\alpha} v_{\perp}^{2} / 2 B_{0}\right)$ is related to the total perturbed distribution function $f_{\alpha}=f_{\alpha}(\mathbf{k}, \epsilon, \mu)$ as

$$
f_{\alpha}=q_{\alpha} \phi \frac{\partial F_{0 \alpha}}{\partial \epsilon}+\exp \left(i L_{\alpha}\right) h_{\alpha} .
$$

The ion and electron gyrokinetic distribution functions are coupled by the quasineutrality equation

$$
\sum_{\alpha} q_{\alpha} \int f_{\alpha} d \mathbf{v}=0
$$

In the gyrokinetic equation for the ion species, we take into account ion-ion collisions using a linearized operator to describe collisions among particles of the same species. As discussed in the details in Ref. 28, the linearized FokkerPlanck collision operator can be factored into two parts: a pitch-angle scattering operator and an operator that describes energy diffusion. Starting from such operator it is then possible to construct a gyrokinetic collision operator in a systematic way (see Ref. 25). We note that an alternate form of the collision operator is discussed in Ref. 29.

Since damping of the plasma flow arises from a $v_{\|} / v$ anisotropy, we simplify our system by neglecting the energy diffusion part of the collision operator, leaving only the pitch-angle scattering part. This simplification also allows some analytical progress to be made. We therefore write the collision operator as

$$
\mathcal{C}\left(f_{i}\right)=\nu_{i}^{D}(v)\left[\mathcal{L}\left(f_{i}\right)+\frac{m_{i} \mathbf{v} \cdot \mathbf{u}}{T_{i}} F_{0 i}\right],
$$

where the collision frequency $\nu_{i}^{D}(v)$ is given by $\nu_{i}^{D}(v)$ $=\nu_{i} \sqrt{8} v_{\mathrm{th}, i}^{3} H\left(v / v_{\mathrm{th}, i}\right) / v^{3}$, with $\nu_{i}=4 \pi n_{e} e^{4} \ln \lambda /\left[\left(2 T_{i}\right)^{3 / 2} m_{i}^{1 / 2}\right]$, $v_{\text {th }, \alpha}=\left(T_{\alpha} / m_{\alpha}\right)^{1 / 2}$,

$$
H(x)=\frac{\exp \left(-x^{2} / 2\right)}{x \sqrt{\pi / 2}}+\left(1-\frac{1}{x^{2}}\right) \operatorname{erf}(x / \sqrt{2}),
$$

and the Lorentz collision operator $\mathcal{L}$ is defined as

$$
\mathcal{L}=\frac{1}{2} \frac{\partial}{\partial \mathbf{v}}\left(\mathbf{I} v^{2}-\mathbf{v v}\right) \frac{\partial}{\partial \mathbf{v}} .
$$

Since the Lorentz scattering operator conserves particles and energy, but does not conserve momentum, we define the vector $\mathbf{u}$ in Eq. (5) as

$$
\mathbf{u}=\frac{\int \mathbf{v} \nu_{i}(v) f_{i} d \mathbf{v}}{\int \nu_{i}(v) m_{i} v^{2} F_{0 i} d \mathbf{v} /\left(3 T_{i}\right)}
$$

so that the collision operator $\mathcal{C}\left(f_{\alpha}\right)$ in Eq. (5) also conserves momentum. Introducing the value for $f_{i}$ given by Eq. (3) into Eq. (8), one obtains

$$
\mathbf{u}_{\perp}=\frac{i \int \nu_{i}^{D}(v) J_{1}\left(k v_{\perp} / \Omega_{i}\right) v_{\perp} h_{i} d \mathbf{v} \mathbf{b} \times \mathbf{k}}{\int \nu_{i}^{D}(v) m_{i} v^{2} F_{0 i} d \mathbf{v} /\left(3 T_{i}\right)} \frac{\mathbf{k}}{k}
$$

and

$$
\mathbf{u}_{\|}=\frac{i \int \nu_{i}^{D}(v) J_{0}\left(k v_{\perp} / \Omega_{i}\right) v_{\|} h_{i} d \mathbf{v}}{\int \nu_{i}^{D}(v) m_{i} v^{2} F_{0 i} d \mathbf{v} /\left(3 T_{i}\right)} \mathbf{b} .
$$

Since we are interested in the damping of potential structures in the perpendicular direction, we assume, for simplicity, that $h\left(v_{\|}\right)=h\left(-v_{\|}\right)$at $t=0$; it follows that $u_{\|}=0$ at all times since the considered gyrokinetic equation and the collision operator do not introduce any asymmetry in $v_{\|}$. Gyroaveraging the collision operator $\mathcal{C}\left(f_{i}\right)$ as shown in Eq. (2) (more details on the calculation are presented in Appendix A), one finds 


$$
\begin{aligned}
\mathcal{I}_{i}\left(f_{i}\right)= & \nu_{i}^{D}(v)\left[\frac{1}{2} \frac{\partial}{\partial \xi}\left(1-\xi^{2}\right) \frac{\partial h_{i}}{\partial \xi}-\frac{v^{2}\left(1+\xi^{2}\right)}{4 \Omega_{i}^{2}} k_{\perp}^{2} h_{i}\right. \\
& \left.-i \frac{m_{i}}{T_{i}} J_{1}\left(\frac{k_{\perp} v_{\perp}}{\Omega_{i}}\right) u_{\perp} v_{\perp} F_{0 \alpha}\right]
\end{aligned}
$$

where $\xi=v_{\|} / v$.

In the collision operator of the electron gyrokinetic equation, we take into account collisions with ions, in addition to electron-electron collisions that can be described as in Eq. (11). The operator for electron-ion collisions can be written as

$$
\mathcal{C}_{e i}=\frac{\sqrt{8} \nu_{e} v_{\text {th }, e}^{3}}{v^{3}} \mathcal{L}\left(f_{e}\right),
$$

where $\nu_{e}=4 \pi n_{e} e^{4} \ln \lambda /\left[\left(2 T_{e}\right)^{3 / 2} m_{e}^{1 / 2}\right]$. Since electron-ion collision does not need to conserve momentum, the total collision operator for electrons becomes

$$
\begin{aligned}
\mathcal{I}_{e}\left(f_{e}\right)= & \nu_{e}^{S}(v) \frac{1}{2}\left[\frac{\partial}{\partial \xi}\left(1-\xi^{2}\right) \frac{\partial h_{e}}{\partial \xi}-\frac{v^{2}\left(1+\xi^{2}\right)}{2 \Omega_{e}^{2}} k_{\perp}^{2} h_{e}\right] \\
& -i \nu_{e}^{D}(v) F_{0 e} J_{1}\left(\frac{k_{\perp} v_{\perp}}{\Omega_{e}}\right) u_{\perp} v_{\perp},
\end{aligned}
$$

where $\nu_{e}^{S}(v)=v_{e} \sqrt{8} v_{\mathrm{th}, e}^{3}\left[1+H\left(v / v_{\mathrm{th}, e}\right)\right] / v^{3}$.

\section{TIME SCALES}

Based on the assumptions that $k_{\perp} \rho_{i} \leqslant 1,\left(\rho_{i}=v_{\mathrm{th}, i} / \Omega_{c i}\right)$, and $T_{e} \sim T_{i}$, the analysis of the electron and ion collision operators leads to the identification of three time scales. The pitch-angle scattering part of the electron collision operator $\nu_{e}^{S}(v) \mathcal{L}\left(h_{e}\right)$ operates on the fastest time scale $\nu_{e} \sim \nu_{i} \sqrt{m_{i} / m_{e}}$ and leads to the isotropization of the electron distribution function. The second time scale of interest is on the order of $\nu_{i}$ and concerns the full ion collision operator [Eq. (11)]. On this time scale, the ion distribution function is isotropized and the potential is damped. The parts of the electron collision operator that arise due to electron FLR and momentum conserving terms, which correspond to the second and third terms on the right-hand side of Eq. (13), are responsible for damping of the flow pattern on the slowest time scale present in the system $\nu_{e}\left(k_{\perp} \rho_{e}\right)^{2} \sim \nu_{i} \sqrt{m_{e} / m_{i}}$. Here we focus on the ion collisional time scale (nonlinear simulations carried out in a $\mathrm{Z}$ pinch, for example, show that this is the typical time scale related to the damping of the flows). Thus, we assume that the electron distribution function is isotropized on a faster time scale than the one of interest and we neglect the damping of the potential due to electron FLR effects and momentum conserving terms. It follows that the total electron collision operator can be neglected, and the electron gyrokinetic equation becomes

$$
\frac{\partial h_{e}}{\partial t}=e \frac{d \phi}{d t} \frac{\partial F_{0 e}}{\partial \epsilon},
$$

where we have made the further assumption $J_{0}\left(k_{\perp} v_{\perp} / \Omega_{e}\right)$ $\simeq 1$ since $k_{\perp} \rho_{e} \ll 1$. Taking the time derivative of the Poisson equation [Eq. (4)] and making use of Eq. (14), one is led to the following quasineutrality constraint:

$$
2 \pi \int J_{0}\left(\frac{k v_{\perp}}{\Omega_{i}}\right) \frac{\partial h_{i}}{\partial t} v^{2} d v d \xi=\frac{e}{T_{i}} \frac{d \phi}{d t} .
$$

Consistent with these assumptions, we present numerical results that consider the gyrokinetic equation for the ions,

$$
\begin{aligned}
\frac{\partial h_{i}}{\partial t}= & -e \frac{d \phi}{d t} \frac{\partial F_{0 i}}{\partial \epsilon} J_{0}\left(\frac{k_{\perp} v_{\perp}}{\Omega_{i}}\right)+\nu_{i}^{D}(v)\left[\frac{1}{2} \frac{\partial}{\partial \xi}\left(1-\xi^{2}\right) \frac{\partial h_{i}}{\partial \xi}\right. \\
& \left.-\frac{v^{2}\left(1+\xi^{2}\right)}{4 \Omega_{i}^{2}} k_{\perp}^{2} h_{i}-i \frac{m_{i}}{T_{i}} J_{1}\left(\frac{k_{\perp} v_{\perp}}{\Omega_{i}}\right) u_{\perp} v_{\perp} F_{0 i}\right]
\end{aligned}
$$

and the Poisson equation [Eq. (15)]. This system of equations describes the damping of the potential due to collisions on the $\nu_{i}$ time scale. We note that the stationary solutions of Eqs. (15) and (16) are given by $h_{i}=0$ and $\phi=0$; thus, we expect that the system evolves in such a way that the level of zonal flows decreases to a negligible value.

We remark that neglecting any of the terms in the collision operator in Eq. (16) can lead to nonphysical evolution of the flows. In the case that the momentum conserving terms are neglected, a nonphysical flow damping on the $\nu_{i}$ time scale is observed for $k \rightarrow 0$. Also, neglecting the Lorentz operator leads to nonphysical consequences: in this case, one observes the zonal flow exponential growth instead of their damping. Such nonphysical effects are discussed in detail in Appendix B.

\section{NUMERICAL RESULTS}

We turn next to the numerical solutions of Eqs. (15) and (16). The numerical implementation of the collision operator in GS2 is described in Ref. 27, while an earlier implementation of FLR effects in GS2 is described in Ref. 30. As a check on the GS2 results, we have also developed an independent gyrokinetic solver based on a simpler numerical algorithm: we discretize velocity space on a rectangular grid and use the trapezoid rule to evaluate the velocity space integrals. The gyrokinetic equations are evolved implicitly in time.

Since the damping of the flows depends on the details of the ion distribution function, it is not possible to provide general damping rates that are completely independent of the initial conditions. In general, it is observed that if the initial distribution function depends sufficiently strongly on $\xi$, the damping of the flows arises mainly from the isotropizing effect of the Lorentz term present in Eq. (16). This damping mechanism is present even for $k \rho_{i} \rightarrow 0$. In the case that the initial distribution function is relatively isotropic, on the other hand, the damping rate of the flows is essentially due to FLR effects.

One can estimate the importance of the Lorentz part of the collision operator with respect to the FLR damping by comparing the second and third terms of the right-hand side of Eq. (16). In particular, defining $\partial_{\xi} h_{i} \sim h_{i} / L_{\xi}$, one has $\partial_{\xi}\left[\left(1-\xi^{2}\right) \partial_{\xi} h_{i}\right] \sim h_{i}\left(1+L_{\xi}\right) / L_{\xi}^{2}$ and $v^{2}\left(1+\xi^{2}\right) /\left(4 \Omega_{i}^{2}\right) k_{\perp}^{2} h_{i}$ $\sim\left(k_{\perp} \rho_{i}\right)^{2} h_{i}$, thus the FLR part of the collision operator dominates over the Lorentz part if $\left(k_{\perp} \rho_{i}\right)^{2} L_{\xi}^{2} /\left(1+L_{\xi}\right)>1$. This is the case of interest in the numerical results that follow, in which we consider a Maxwellian gyrocenter distribution 

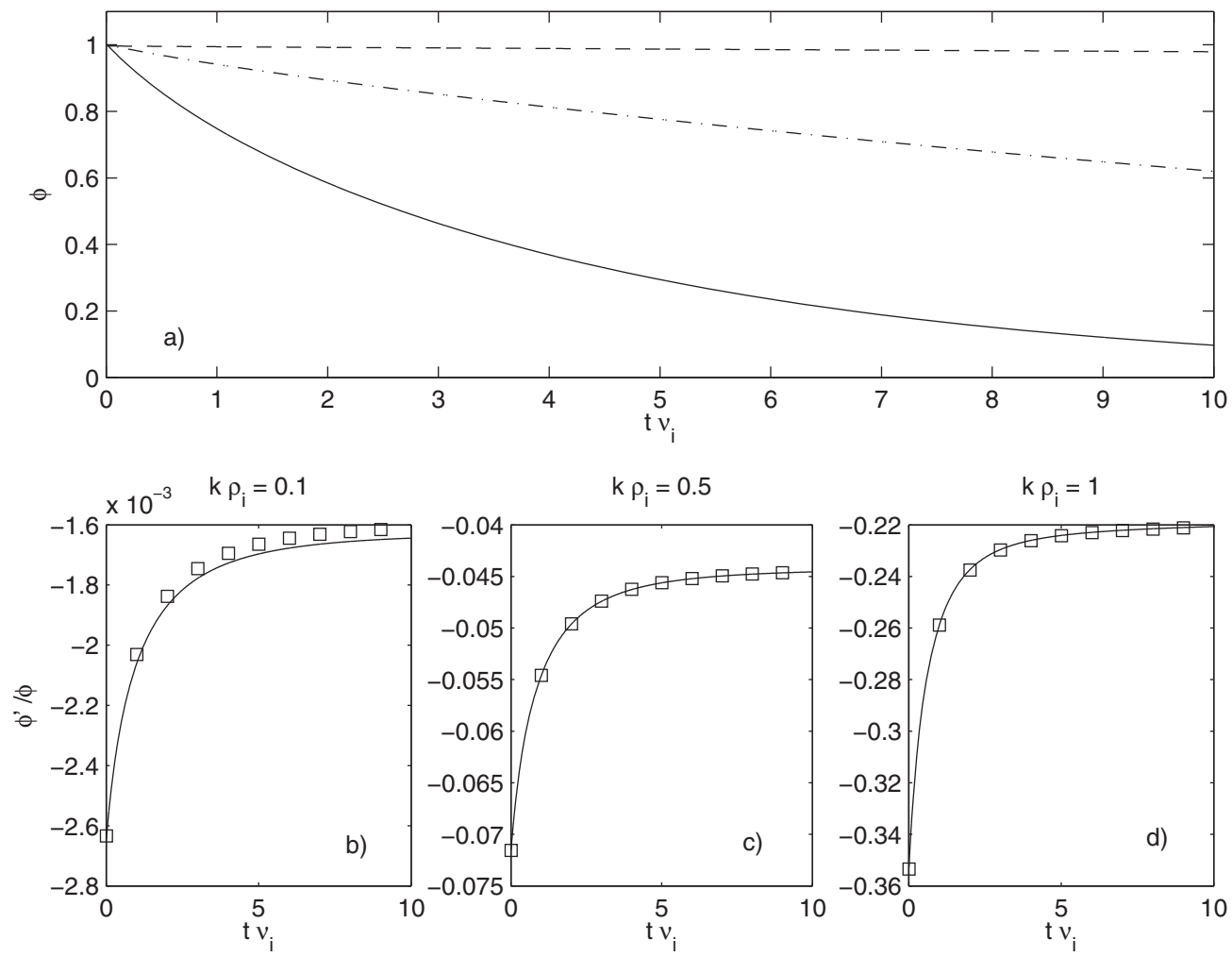

FIG. 1. Damping of the potential for $k \rho_{i}=0.1$ (dashed line), $k \rho_{i}=0.5$ (dashed-dotted line), and $k \rho_{i}=1$ (solid line) as a function of time (a). The damping rate, $(d \phi / d t) / \phi$, is plotted for (b) $k \rho_{i}=0.1$, (c) $k \rho_{i}=0.5$, and (d) $k \rho_{i}=1$. The squares show the damping rate evaluated by the independent gyrokinetic solver.

function as an initial distribution function, i.e., $\left.h_{i}\right|_{t=0}$ $=n_{s} \exp \left[-m_{i} v^{2} /\left(2 T_{i}\right)\right] /\left(2 \pi v_{\mathrm{th}, i}^{2}\right)^{3 / 2}$. We note that the solution is independent of $n_{s}$ and $v_{\text {th }, i}$.

In Fig. 1(a) we show the typical evolution of the potential as a function of time for $k \rho_{i}=0.1,0.5,1$. We also plot the damping rates in Figs. 1(b)-1(d) as function of time for the same values of $k \rho_{i}$. Figures $1(\mathrm{~b})-1(\mathrm{~d})$ show that the damping is faster at $t=0$ [we denote the value of the damping rate at $t=0$ with $\left.\gamma_{\max }^{0}(k)\right]$, and it becomes slower at later times. This is due to the fact that the distribution function $h_{i}$ is depleted in the phase space region where the damping is the strongest. The consequence is that, at later times, the weakly damped phase space regions of $h_{i}$ dominate, leading to an overall decrease of the damping rate with time. The GS2 code and the independent gyrokinetic solver show excellent agreement, particularly in the case of higher $k \rho_{i}$ in which the numerical solution of the gyrokinetic equations is less demanding. This increases the reliability of the proposed solutions and confirms the correctness of the implementation of the GS2 collision operator.

The dependence of the flow damping rate on the flow scale length is shown in Fig. 2, where the damping rate $\gamma_{\max }^{0}$ is plotted as a function of $k \rho_{i}$. Figure 2(a) shows that $\gamma_{\max }^{0} \rightarrow 0$ for $k \rightarrow 0$ (a rigorous proof of this property is provided in Appendix B). In Fig. 2(b), we plot $\gamma_{\max }^{0}$, normalized to $k^{2} \nu_{i}$, showing that $\gamma_{\max }^{0} \propto k^{2}$ for $k \rightarrow 0$, in particular, $\gamma_{\max }^{0}$ $\simeq-0.265 k^{2} \nu_{i}$ for $k \rightarrow 0$. By assuming $v \sim v_{\text {th }, i}$, one can estimate the term dominating the $h_{i}$ damping due to collisional FLR effects, i.e., the second term on the right-hand side of Eq. (16), as $\nu_{i}^{D}(v) v^{2}\left(1+\xi^{2}\right) k_{\perp}^{2} h_{i} /\left(4 \Omega_{i}\right) \simeq 0.3 \nu_{i}\left(k_{\perp} \rho_{i}\right)^{2} h_{i}$, in good agreement with the numerical findings. The momentum conserving terms and kinetic effects are responsible for the discrepancies from this simple $k^{2}$ scaling, in particular a faster damping than the one given by the $k^{2}$ proportionality is obtained for $k \rho_{i} \sim 1$.

The fact that the damping rate vanishes for $k \rho_{i} \rightarrow 0$ is related to the origin of the perpendicular flow damping: the $\xi$ dependence of the initial distribution function, which is smoothed out by the collision operator. This dependence appears in the $\exp \left(i L_{\alpha}\right)$ term in Eq. (3) since $L_{\alpha}$ $\propto v \sqrt{1-\xi^{2}} k_{\perp} / \Omega_{\alpha}$. Thus, the damping is fundamentally related to FLR effects and, as expected, vanishes in the limit of long wavelength. As shown in Appendix B, in the case that the momentum conserving terms are neglected, then one observes the nonphysical presence of finite flow damping on the $\nu_{i}$ time scale for $k \rightarrow 0$.

The shape of the ion distribution function $h_{i}$ is plotted in Fig. 3 for different values of $k \rho_{i}$. As shown in Fig. 3, for all the values of $k \rho_{i}, h_{i}$ decreases more for $|\xi| \geqslant 0.7$ and the most relevant changes in the distribution function are localized at $v / v_{\text {th }} \simeq 1.5$. In fact, the $h_{i}$ function is depleted in the phase space region where the second term on the right-hand side of Eq. (16), proportional to $\nu_{i}^{D}(v) v^{2}\left(1+\xi^{2}\right) h_{i}$, is the largest.

\section{CONCLUSIONS}

In the present paper, we have focused attention on the damping of seeded ion Larmor radius scale flows perpendicular to the magnetic field, which is caused by the combined action of ion collisions and FLR effects. As shown by 

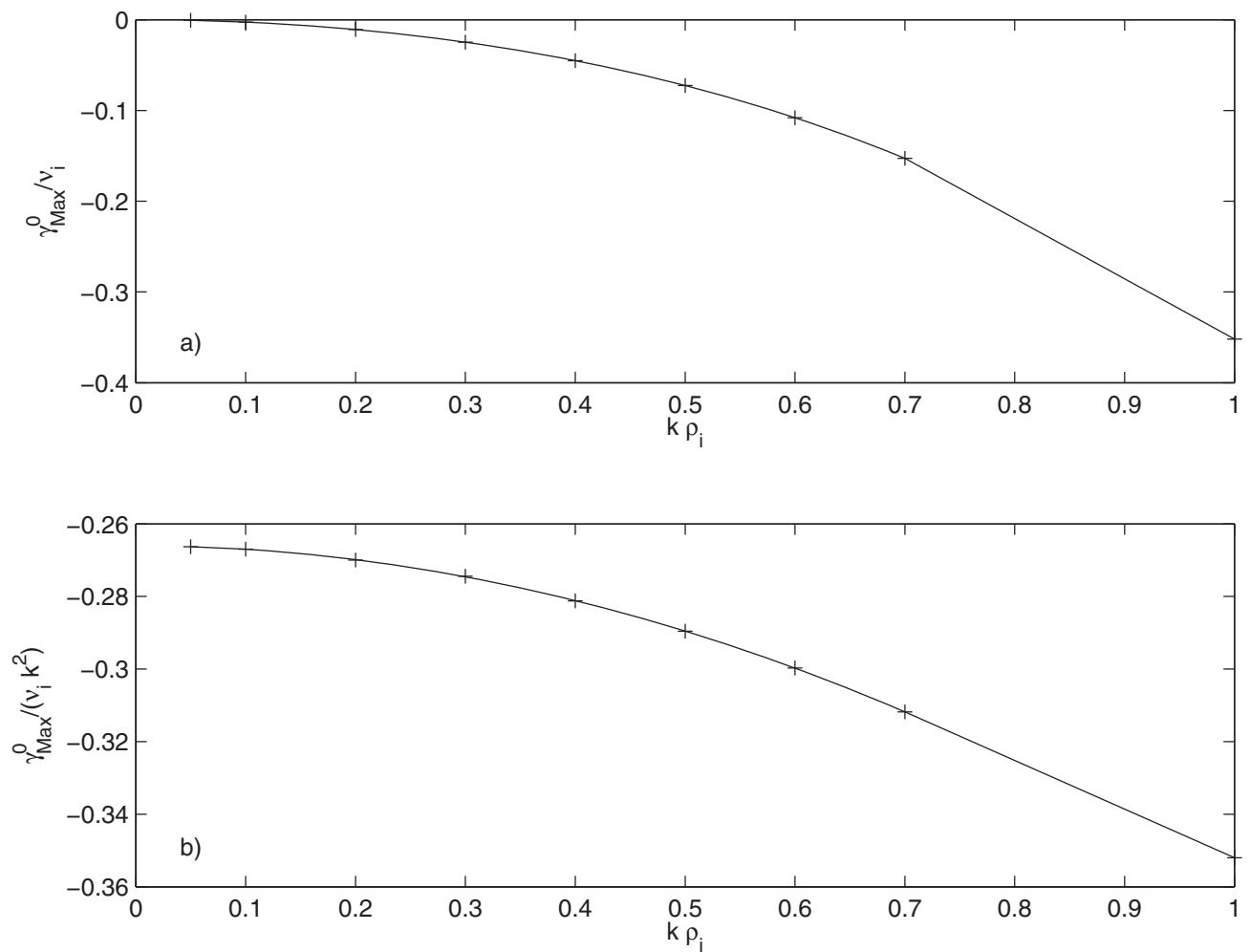

FIG. 2. The damping rate at $t=0, \gamma_{\max }^{0}$, is plotted as a function of $k \rho_{i}$, and normalized to (a) $\nu_{i}$ and (b) $\nu_{i}\left(k \rho_{i}\right)^{2}$.

recent gyrokinetic simulations of turbulence in the $\mathrm{Z}$ pinch and dipolar configurations, we believe this mechanism is particularly important in systems without significant trapped particle populations, in which collisions can be the main source of perpendicular flow damping. Our numerical solutions of the gyrokinetic equations in a simple homogeneous system show that the flow damping vanishes in the limit of long wavelengths as $\left(k \rho_{i}\right)^{2}$. The most relevant changes in the ion distribution function that arise during the damping process are localized at $v / v_{\text {th }} \simeq 1.5$ and $|\xi| \geqslant 0.7$. Finally, as described in Appendix B, we find that a proper account of FLR effects in the collision operator is essential to correctly describe the damping mechanism. In particular, the neglect of the momentum conserving terms leads to nonphysical zonal flow damping in the limit of $k \rightarrow 0$.

The numerical results presented here have been obtained by using the GS2 gyrokinetic code and have been benchmarked against an independent numerical code. This increases the reliability of the proposed solutions and confirms the correctness of the implementation of the GS2 collision operator. The numerical results shown here can indeed be used as a benchmark test for the implementation of FLR effects in the collision operator of gyrokinetic codes, in addition to the suite of tests that are described in Ref. 27. We briefly summarize the hypothesis under which the proposed benchmark tests should be carried out. The gyrokinetic system can be reduced to Eqs. (15) and (16) if the following hypothesis are satisfied: (i) curvature, equilibrium gradients, parallel dynamics, and nonlinear terms are neglected; (ii) $k_{\perp} \rho_{e} \ll 1$; and (iii) the energy diffusion part of the collision operator is neglected. In order to solve Eqs. (15) and (16) we have assumed that $h_{i}$ is a Maxwellian distribution function at time $t=0$, i.e., $\left.h_{i}\right|_{t=0}=n_{s} \exp \left[-m_{i} v^{2} /\left(2 T_{i}\right)\right] /\left(2 \pi v_{\text {th }, i}^{2}\right)^{3 / 2}$ (we note that the numerical solution is independent of $n_{s}$ and $\left.v_{\text {th }, i}\right)$. The damping rate of the zonal flow is shown in Figs. 1 and 2, while Fig. 3 shows how the ion distribution function is affected by the collision term.

We finally note that the present study concerns a physical system in which the role of FLR collisional effects in zonal flow damping is enhanced with respect to what is expected in more realistic and complex situations. We have in fact considered a system where the effect of self-consistent turbulence, curvature, and particle trapping is not present, and the gyrocenter distribution functions are Maxwellian. In this configuration, the only damping mechanism of $\mathbf{E} \times \mathbf{B}$ zonal flows can only be provided by FLR collisional effects. The importance of the FLR collisional terms in more realistic conditions remains to be assessed.

\section{ACKNOWLEDGMENTS}

Financial support from the NASA Sun-Earth Connection Program under Grant No. NNG05GJ70G, from the DoE Center for Multiscale Plasma Dynamics, from the DoE/ EPSCoR/CICART, and from the "Fonds National Suisse de la Recherche Scientifique" is acknowledged.

\section{APPENDIX A: DERIVATION OF THE COLLISION OPERATOR FOR GYROKINETIC EQUATIONS}

We derive the collision operator expressed in Eq. (11) with $\mathcal{C}\left(f_{\alpha}\right)$ defined in Eq. (5). Using the velocity variables $v$, $\xi=v_{\|} / v$, and $\zeta$ (the gyrophase angle), the collision operator (5) can be written as 
a)

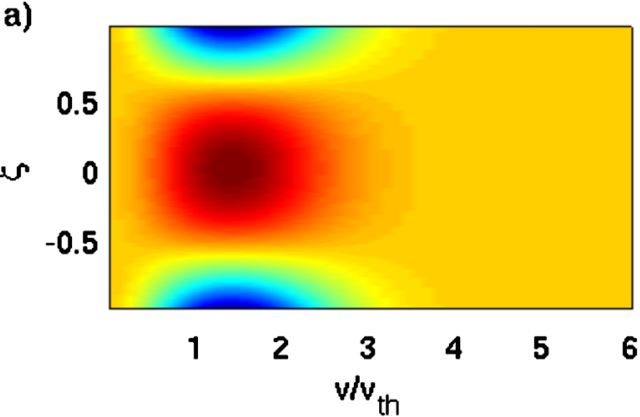

b)

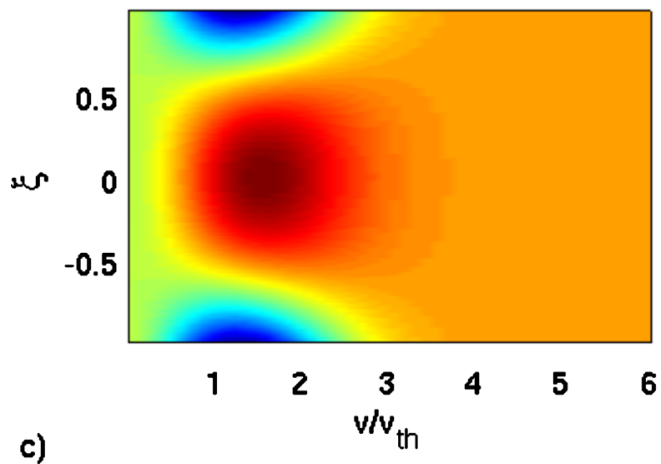

c)

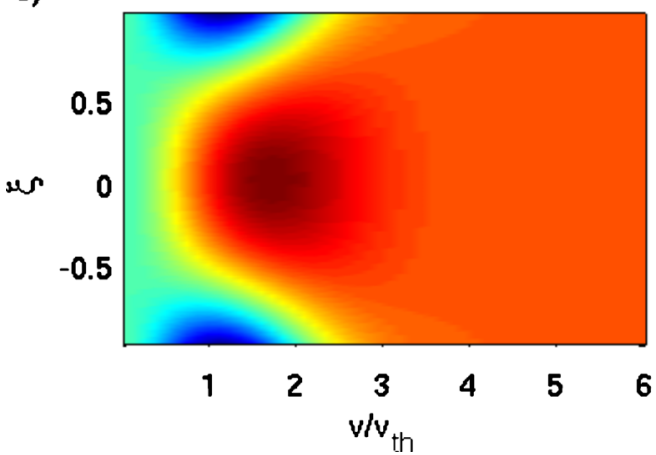

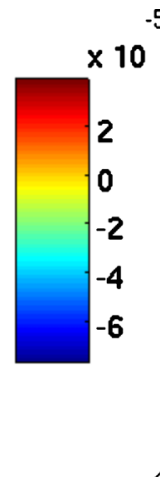
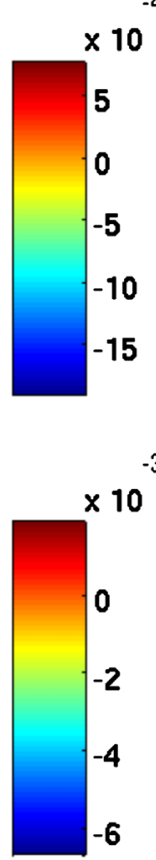

$-5$

FIG. 3. (Color online) The difference between the initial distribution function $h_{i}=F_{0}$ and the normalized distribution function $h_{i} / \int h_{i} d \mathbf{v}$ at time $t=10$ is plotted for (a) $k \rho_{i}=0.1$, (b) $k \rho_{i}=0.5$, and $k \rho_{i}=1$ as a function of $v / v_{\text {th }}$ and $\xi=v_{\|} / v$.

$$
\mathcal{C}\left(f_{\alpha}\right)=\nu_{\alpha}(\epsilon)\left\{\frac{\partial}{\partial \xi}\left[\left(1-\xi^{2}\right) \frac{\partial f_{\alpha}}{\partial \xi}\right]-\frac{1}{1-\xi^{2}} \frac{\partial^{2} f_{\alpha}}{\partial \zeta^{2}}\right\}
$$

Since $F_{0}=F_{0}(v)$ and $h_{\alpha}$ is defined according to Eq. (3), it follows that $\mathcal{C}\left(f_{\alpha}\right)=\mathcal{C}\left[h_{\alpha} \exp (i L)\right]$, and the collision integral $\mathcal{I}_{\alpha}\left(f_{\alpha}\right)$ can be written as

$$
\begin{aligned}
\mathcal{I}_{\alpha}\left(f_{\alpha}\right)= & \nu_{\alpha}(\epsilon) \int_{-\pi}^{\pi} \frac{d \zeta}{2 \pi} \exp (-i L) \\
& \times\left\{\frac{\partial}{\partial \xi}\left[\left(1-\xi^{2}\right) \frac{\partial h_{\alpha} \exp (i L)}{\partial \xi}\right]\right. \\
& \left.-\frac{1}{1-\xi^{2}} \frac{\partial^{2} h_{\alpha} \exp (i L)}{\partial \xi^{2}}+\frac{m_{\alpha} \mathbf{v} \cdot \mathbf{u}}{T_{0 \alpha}} F_{0 \alpha}\right\}
\end{aligned}
$$

In order to perform the integration in $\zeta$, we note that

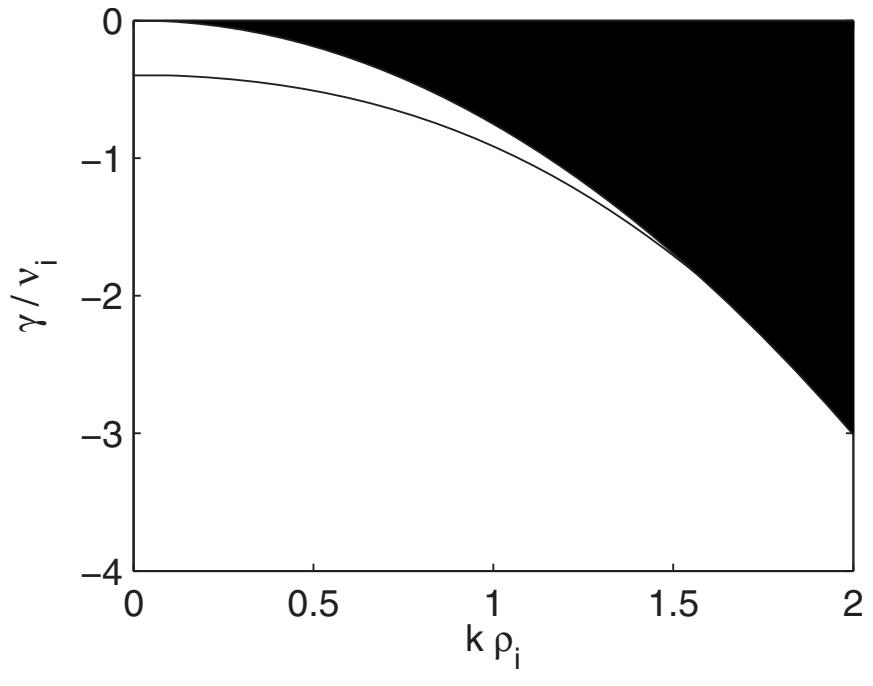

FIG. 4. Values of $\gamma$ that are solutions of Eq. (B4) as a function of $k \rho_{i}$.

$$
L_{\alpha}=\frac{\mathbf{v}_{\perp} \times \mathbf{b} \cdot \mathbf{k}}{\Omega_{\alpha}}=\frac{v \sqrt{1-\xi^{2}}\left(\mathbf{e}_{1} \cos \zeta+\mathbf{e}_{2} \sin \zeta\right) \times \mathbf{b} \cdot \mathbf{k}}{\Omega_{\alpha}},
$$

where $\mathbf{e}_{1}$ and $\mathbf{e}_{2}$ are two versors perpendicular to the magnetic field and perpendicular to each other. Without any loss of generality, we take $\mathbf{e}_{2}$ to be parallel to $\mathbf{k}_{\perp}$ so that

$$
L_{\alpha}=\frac{v \sqrt{1-\xi^{2}}}{\Omega_{\alpha}} k_{\perp} \cos \zeta
$$

We start by considering the first term on the right-hand side of Eq. (A2),

$$
\begin{aligned}
\int_{-\pi}^{\pi} & \frac{d \zeta}{2 \pi} \exp \left(-i L_{\alpha}\right) \frac{\partial}{\partial \xi}\left(1-\xi^{2}\right) \frac{\partial\left[h_{\alpha} \exp \left(i L_{\alpha}\right)\right]}{\partial \xi} \\
= & h_{\alpha} \int_{-\pi}^{\pi} \frac{d \zeta}{2 \pi} \exp \left(-i L_{\alpha}\right) \frac{\partial}{\partial \xi}\left[\left(1-\xi^{2}\right) \frac{\partial \exp \left(i L_{\alpha}\right)}{\partial \xi}\right] \\
& +\int_{-\pi}^{\pi} \frac{d \zeta}{2 \pi} \exp \left(-i L_{\alpha}\right) \frac{\partial\left[\left(1-\xi^{2}\right) h_{\alpha}\right]}{\partial \xi} \frac{\partial \exp \left(i L_{\alpha}\right)}{\partial \xi} \\
& +\int_{-\pi}^{\pi} \frac{d \zeta}{2 \pi} \exp \left(-i L_{\alpha}\right) \frac{\partial \exp \left(i L_{\alpha}\right)}{\partial \xi}\left(1-\xi^{2}\right) \frac{\partial h_{\alpha}}{\partial \xi} \\
& +\frac{\partial}{\partial \xi}\left(1-\xi^{2}\right) \frac{\partial h_{\alpha}}{\partial \xi} .
\end{aligned}
$$

Since

$$
\frac{\partial \exp \left(i L_{\alpha}\right)}{\partial \xi}=\frac{i \xi}{\sqrt{1-\xi^{2}}} v k_{\perp} \cos \zeta \exp \left(i L_{\alpha}\right)
$$

the second and third terms in the sum in the right-hand side of Eq. (A5) vanish when they are gyroaveraged, and the first term in Eq. (A5) gives 


$$
\begin{aligned}
& h_{\alpha} \int_{-\pi}^{\pi} \frac{d \zeta}{2 \pi} \exp \left(-i L_{\alpha}\right) \frac{\partial}{\partial \xi}\left(1-\xi^{2}\right) \frac{\partial \exp \left(i L_{\alpha}\right)}{\partial \xi} \\
& \quad=h_{\alpha} \int_{-\pi}^{\pi} \frac{d \zeta}{2 \pi} \frac{\partial}{\partial \xi} \frac{i v k_{\perp}}{\Omega_{\alpha}} \sqrt{1-\xi^{2}} \xi \cos \zeta \\
& =-h_{\alpha} \int_{-\pi}^{\pi} \frac{d \zeta}{2 \pi} \xi^{2} \frac{v^{2} k_{\perp}^{2}}{\Omega_{\alpha}^{2}} \cos ^{2} \zeta \\
& =-\frac{1}{2} h_{\alpha} \xi^{2} \frac{v^{2} k_{\perp}^{2}}{\omega_{\alpha}^{2}} .
\end{aligned}
$$

The second term in Eq. (A2) can also be evaluated as

$$
\begin{aligned}
\int_{-\pi}^{\pi} \frac{d \zeta}{2 \pi} \exp \left(-i L_{\alpha}\right) \frac{1}{1-\xi^{2}} \frac{\partial^{2} h_{\alpha} \exp \left(i L_{\alpha}\right)}{\partial \zeta^{2}} \\
=h_{\alpha} \int_{-\pi}^{\pi} \frac{d \zeta}{2 \pi} \exp \left(-i L_{\alpha}\right) \frac{1}{1-\xi^{2}} \frac{\partial}{\partial \zeta} \\
\quad \times\left[i \frac{v k_{\perp} \sqrt{1-\xi^{2}}}{\Omega_{\alpha}} \sin \zeta \exp \left(i L_{\alpha}\right)\right] \\
=h_{\alpha} \int_{-\pi}^{\pi} \frac{d \zeta}{2 \pi}\left(i \frac{k_{\perp} v}{\Omega_{\alpha}}\right)^{2} \sin ^{2} \zeta=-\frac{1}{2} \frac{k_{\perp}^{2} v^{2}}{\Omega_{\alpha}^{2}} h_{\alpha} .
\end{aligned}
$$

The gyroangle integrations present in the momentum conserving term can be evaluated in a similar way. Considering the result reported in Eqs. (A5), (A7), and (A8), and the gyroangle integrations for the momentum conserving terms, one obtains the collision operator reported in Eq. (11).

\section{APPENDIX B: NONPHYSICAL RESULTS FROM SIMPLIFIED COLLISION OPERATORS}

Neglecting any of the terms in the collision operator of Eq. (16) can lead to a nonphysical evolution of the flow pattern. In this appendix we discuss two examples.

\section{Neglecting the momentum conserving term}

For simplicity, we consider an ion distribution function that does not depend on $\xi$ so that the Lorentz part of the collision operator can be neglected, and we study the effect of neglecting the momentum conserving term in the gyrokinetic equation. With this assumption, the collision operator may be expressed as

$$
\mathcal{I}_{i}\left(f_{i}\right)=-\nu_{i}^{D} \frac{v^{2}\left(1+\xi^{2}\right)}{4 \Omega_{i}^{2}} k_{\perp}^{2} h_{i}=-\nu_{i}^{T}\left(1+\xi^{2}\right) h_{i},
$$

where the function $\nu_{i}^{T}=\nu_{i} \sqrt{8} v_{\mathrm{th}, i}^{3} k_{\perp}^{2} H(v) /\left(4 v \Omega_{i}^{2}\right)$ is such that $\nu_{i}^{T}(v)=2 \nu_{i}\left(k_{\perp} \rho_{i}\right)^{2} /(3 \sqrt{\pi})=\nu_{0}$ for $v \rightarrow 0$ and $\nu_{i}^{T}(v) \sim 1 / v$ for $v \rightarrow \infty$. We study the normal modes in the system, assuming that the potential $\phi$ and the ion distribution function $h_{i}$ have a temporal dependence $\propto \exp (\gamma t)$. The general evolution of the system is given by the superposition of the normal modes excited by the initial conditions. From Eq. (16), the equation for the normal modes is

$$
\left[\gamma+\nu_{i}^{T}\left(1+\xi^{2}\right)\right] h_{i}=e F_{0 i} J_{0} \gamma \phi / T_{i}
$$

with the solution

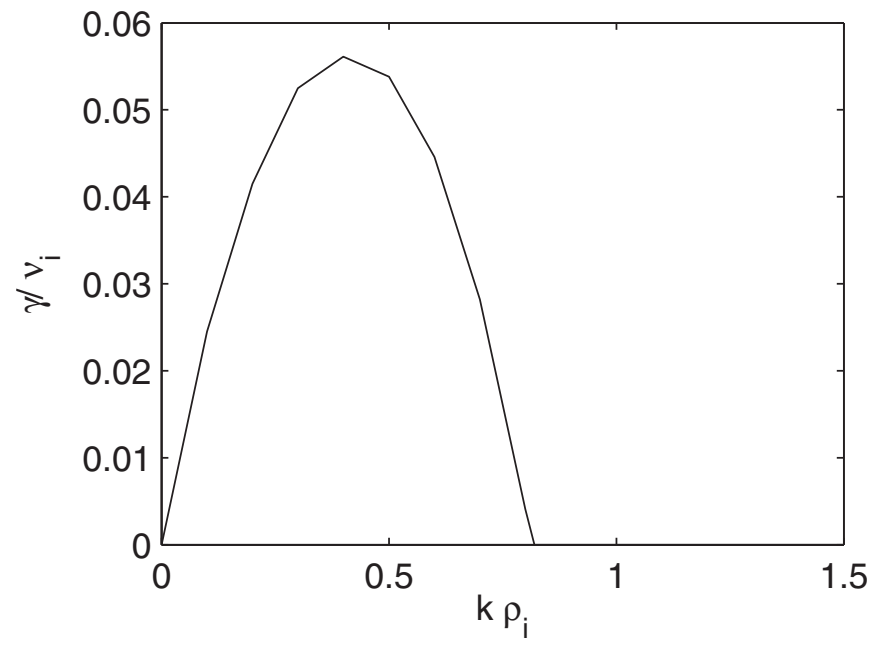

FIG. 5. Growth rate observed in the solution of the gyrokinetic equation, if the Lorentz collision operator is neglected, as a function of $k \rho_{i}$.

$$
h_{i}=\mathcal{P} \frac{\gamma e F_{0 i} J_{0} \phi / T_{i}}{\gamma+\nu_{i}^{T}\left(1+\xi^{2}\right)}+\delta\left[\gamma+\nu_{i}^{T}\left(1+\xi^{2}\right)\right] g(v),
$$

where $\mathcal{P}$ indicates that the integration in velocity space is performed according to the Cauchy principal value and $g(v)$ is an arbitrary function. Introducing Eq. (B3) into Poisson equation [Eq. (15)], one obtains the following eigenvalue equation:

$$
\begin{aligned}
& \mathcal{P} \int \frac{\gamma e F_{0 i} J_{0}^{2} \phi / T_{i}}{\gamma+\nu_{i}^{T}\left(1+\xi^{2}\right)} d \mathbf{v}+\int \delta\left[\gamma+\nu_{i}^{T}\left(1+\xi^{2}\right)\right] g(v) d \mathbf{v} \\
& \quad=\frac{e}{T_{i}} \phi .
\end{aligned}
$$

Equation (B4) admits three sets of solutions. The first set concerns the values of $\gamma$ such that $\gamma \neq\left[\nu_{i}^{T}\left(1+\xi^{2}\right)\right]$ for all $v$ and for all $\xi$ (i.e., $\gamma<-2 \nu_{0}$ ) that obey

$$
\int \frac{\gamma e F_{0} J_{0}^{2} / T_{i}}{\gamma+\nu_{i}^{T}\left(1+\xi^{2}\right)} d \mathbf{v}=1 .
$$

In this case, $h_{i}$ in Eq. (B3) is given by $h=\gamma e F_{0} J_{0} \phi /[\gamma$ $\left.+\nu_{i}^{T}\left(1+\xi^{2}\right)\right] T_{i}$. It is possible to show that only $\gamma \in \mathfrak{R}$ is a solution of Eq. (B5) (i.e., there are no oscillatory solutions). The second set of solutions is found for $\phi=0$ and $\gamma$ such that a value of $v$ and $\xi$ exist for which $\gamma=-\nu_{i}^{T}\left(1+\xi^{2}\right)$ (i.e., $-2 \nu_{0}$ $<\gamma<0)$. In this case, the function $g(v)$ satisfies the equation

$$
\int \delta\left[\gamma+\nu(v)\left(1+\xi^{2}\right)\right] g(v) d \mathbf{v}=0,
$$

and an infinite set of solutions $g(v)$ exists for each value of $\gamma$. This set of solutions does not show any signature on the damping of the flow pattern. The third set of solutions have, once again, $\gamma$ such that a value of $v$ and $\xi$ exist for which $\gamma=-\nu_{i}^{T}\left(1+\xi^{2}\right)$ (i.e., $-2 \nu_{0}<\gamma<0$ ), but $\phi \neq 0$ and $g(v) \propto \phi$; thus, solutions of the third kind have a signature on the flow damping. For each $\gamma$ there is an infinite set of solutions $g(v)$ that satisfy Eq. (B4) in the following form: 


$$
\mathcal{P} \int \frac{\gamma F_{0} J_{0}}{\gamma+\nu_{i}^{D}\left(1+\xi^{2}\right)} d \mathbf{v}+\int \delta\left[\gamma+\nu(v)\left(1+\xi^{2}\right)\right] g(v) d \mathbf{v}=1
$$

In Fig. 4 we show the values of $\gamma$ that are solutions of Eq. (B4). Besides the shaded area that represents the values of $0<\gamma<2 \nu_{0}$, a solution of Eq. (B5) is found for $k \rho_{i} \lesssim 2.1$, denoted with $\gamma_{s}$. It follows that the damping rate of the potential tends to a finite value for $k \rightarrow 0$. This value can be analytically evaluated: Eq. (B5) leads to

$$
\begin{aligned}
& \int \frac{\left[\gamma_{s}+\nu_{i}^{T}\left(1+\xi^{2}\right)\right] e F_{0} J_{0}^{2} / T_{i}}{\gamma_{s}+\nu_{i}^{T}\left(1+\xi^{2}\right)} d \mathbf{v} \\
& -\int \frac{\nu_{i}^{T}\left(1+\xi^{2}\right) e F_{0} J_{0}^{2} / T_{i}}{\gamma_{s}+\nu_{i}^{T}\left(1+\xi^{2}\right)} d \mathbf{v}=1,
\end{aligned}
$$

which can be rewritten as

$$
\Gamma_{0}\left(k^{2} \rho_{i}^{2}\right)-\frac{1}{\gamma_{s}} \int \nu_{i}^{T}\left(1+\xi^{2}\right) F_{0} d \mathbf{v}=1,
$$

where $\Gamma_{0}(x)=\exp (-x) I_{0}(x)$ with $I_{0}(x)$ as the modified Bessel function, and where we have kept only the first order terms in $\left(k_{\perp} \rho_{i}\right)^{2}$. It follows that

$$
\lim _{k \rightarrow 0} \gamma_{s}=\lim _{k \rightarrow 0} \frac{\int \nu_{i}^{T}\left(1+\xi^{2}\right) F_{0} d \mathbf{v}}{\Gamma_{0}-1} \simeq 0.401 \nu_{i} .
$$

This is an unphysical result since FLR effects are seen to produce a finite damping rate even in the limit of $k \rho_{i} \rightarrow 0$. On the other hand, we can show that the damping rate vanishes for $k \rightarrow 0$ in the presence of the momentum conserving terms. We consider the kinetic equation where the momentum conserving term is included

$$
\gamma h_{i}=e \gamma F_{0} J_{0} \phi-\nu_{i}^{T}\left(1+\xi^{2}\right) h_{i}+\alpha \int \beta h_{i} d \mathbf{v}
$$

with $\alpha=3 \nu_{i}^{D} J_{1} v_{\perp} F_{0} / \int \nu_{i}^{D} v^{2} F_{0} d \mathbf{v}$ and $\beta=\nu_{i}^{D} J_{1} v_{\perp}$. Let us suppose that a finite $\gamma_{s}$ exists for $k \rightarrow 0$. The solution of Eq. (B11) is

$$
h_{i}=\frac{e \gamma F_{0} J_{0} \phi / T_{i}+\alpha \int \beta h_{i} d \mathbf{v}}{\gamma+\nu_{i}^{T}\left(1+\xi^{2}\right)} .
$$

It is possible to express the value of $\int \beta h_{i}$ as a function of $\phi$ if one multiplies Eq. (B12) by $\beta$ and integrates over $d \mathbf{v}$,

$$
\begin{aligned}
\int \beta h_{i} d \mathbf{v} & =\frac{\int e \gamma F_{0} J_{0} \beta \phi /\left[\gamma+\nu_{i}^{T}\left(1+\xi^{2}\right)\right] d \mathbf{v}}{1-\int \alpha \beta /\left[\gamma+\nu_{i}^{T}\left(1+\xi^{2}\right)\right] d \mathbf{v}} \\
& \simeq \frac{\gamma e \phi \int F_{0} J_{0} \beta d \mathbf{v}}{\gamma}=\delta \phi
\end{aligned}
$$

for $k \rho_{i} \ll 1$, with $\delta=e \int F_{0} J_{0} \beta d \mathbf{v}$. Using this result in Eq. (B12), one obtains

$$
h_{i}=\frac{e \gamma F_{0} J_{0} \phi+\alpha \delta \phi}{\gamma+\nu_{i}^{T}\left(1+\xi^{2}\right)} .
$$

This can be inserted into the Poisson equation, leading to the following expression for the damping rate:

$$
\gamma=\frac{1}{1-\Gamma_{0}} \int\left[J_{0} \alpha \delta-\nu_{i}^{T}\left(1+\xi^{2}\right) e F_{0} J_{0}^{2}\right] d \mathbf{v}
$$

in the case of $k \rho_{i} \ll 1$. Since $J_{1}(x) \sim x / 2$ and $J_{0} \sim 1$ for $x \rightarrow 0$, we have

$$
\begin{gathered}
\int J_{0} \alpha \delta d \mathbf{v}=3 \frac{\left(\int F_{0} J_{0} J_{1} \nu_{i}^{D} v_{\perp} d \mathbf{v}\right)^{2}}{\int \nu_{i}^{D} F_{0} v^{2} d \mathbf{v}} \simeq \frac{2}{3}\left(k \rho_{i}\right)^{2} I \\
\int \nu_{i}^{T}\left(1+\xi^{2}\right) e F_{0} J_{0}^{2} d \mathbf{v} \simeq\left(k \rho_{i}\right)^{2} \int \nu_{i}^{D} v^{2}\left(1+\xi^{2}\right) e F_{0} d \mathbf{v} / 4 \\
=\frac{2}{3}\left(k \rho_{i}\right)^{2} I,
\end{gathered}
$$

where $I=2 \pi \int_{0}^{\infty} \nu_{i}^{D} F_{0} v^{4} d v$. This shows that no finite damping due to FLR effects is present for $k \rho_{i} \rightarrow 0$ if the momentum conserving term is accurately taken into account.

\section{Neglecting the Lorentz collision operator}

If one neglects the Lorentz term and considers the collision operator in the form of Eq. (B11), the solution of the gyrokinetic equation shows that the potential increases, instead of being damped out. In Fig. 5 we plot the growth rate of the modes if the Lorentz collision operator is neglected. When the Lorentz term is included, the positive growth rate disappears for all the values of $k \rho_{i}$ considered.

${ }^{1}$ P. H. Diamond, S.-I. Itoh, K. Itoh, and T. S. Hahm, Plasma Phys. Controlled Fusion 47, R35 (2005).

${ }^{2}$ K. Itoh, S.-I. Itoh, P. H. Diamond, T. S. Hahm, A. Fujisawa, G. R. Tynan, M. Yagi, and Y. Nagashima, Phys. Plasmas 13, 055502 (2006).

${ }^{3}$ W. Dorland and G. Hammett, Phys. Fluids B 5, 812 (1993).

${ }^{4}$ Z. Lin, T. Hahm, W. Lee, W. Tang, and R. White, Science 281, 1835 (1998).

${ }^{5}$ J. Candy and R. Waltz, Phys. Rev. Lett. 91, 045001 (2003).

${ }^{6}$ S. E. Parker, J. J. Kohut, Y. Chen, Z. Lin, F. L. Hinton, and W. W. Lee, AIP Conf. Proc. 871, 193 (2006).

${ }^{7}$ D. R. Ernst, P. T. Bonoli, and P. J. Catto, Phys. Plasmas 11, 2637 (2004).

${ }^{8}$ J. Lang, Y. Chen, and S. E. Parker, Phys. Plasmas 14, 082315 (2007).

${ }^{9}$ P. Ricci, B. N. Rogers, and W. Dorland, Phys. Rev. Lett. 97, 245001 (2006).

${ }^{10}$ S. Kobayashi, B. N. Rogers, and W. Dorland, Phys. Rev. Lett. 103, 055003 (2009)

${ }^{11}$ B. N. Rogers, W. Dorland, and M. Kotschenreuther, Phys. Rev. Lett. 85, 5336 (2000).

${ }^{12}$ F. Hinton and M. Rosenbluth, Plasma Phys. Controlled Fusion 41, A653 (1999).

${ }^{13}$ E.-J. Kim, C. Holland, and P. H. Diamond, Phys. Rev. Lett. 91, 075003 (2003).

${ }^{14}$ Y. Xiao, P. J. Catto, and K. Moving, Phys. Plasmas 14, 032302 (2007).

${ }^{15}$ Y. Xiao, P. J. Catto, and W. Dorland, Phys. Plasmas 14, 055910 (2007).

${ }^{16}$ S. Braun, P. Helander, E. A. Belli, and J. Candy, Plasma Phys. Controlled Fusion 51, 065011 (2009).

${ }^{17}$ Z. Lin, T. S. Hahm, W. W. Lee, W. M. Tang, and P. H. Diamond, Phys. Rev. Lett. 83, 3645 (1999).

${ }^{18}$ P. Xanthopoulos, F. Merz, T. Gorler, and F. Jenko, Phys. Rev. Lett. 99, 035002 (2007).

${ }^{19}$ G. Dif-Pradalier, V. Grandgirard, Y. Sarazin, X. Garbet, and Ph. Ghendrih, Phys. Rev. Lett. 103, 065002 (2009).

${ }^{20}$ G. L. Falchetto and M. Ottaviani, Phys. Rev. Lett. 92, 025002 (2004).

${ }^{21}$ T. M. Antonsen and B. Lane, Phys. Fluids 23, 1205 (1980).

${ }^{22}$ A. Brizard and T. S. Hahm, Rev. Mod. Phys. 79, 421 (2007).

${ }^{23}$ M. Kotschenreuther, G. Rewoldt, and W. M. Tang, Comput. Phys. Commun. 88, 128 (1995). 
${ }^{24}$ W. Dorland, F. Jenko, M. Kotschenreuther, and B. N. Rogers, Phys. Rev. Lett. 85, 5579 (2000).

${ }^{25}$ I. G. Abel, M. Barnes, S. C. Cowley, W. Dorland, and A. A. Schekochihin, Phys. Plasmas 15, 122509 (2008).

${ }^{26}$ A. A. Schekochihin, S. C. Cowley, W. Dorland, G. W. Hammett, G. G. Howes, E. Quataert, and T. Tatsuno, Astrophys. J., Suppl. Ser. 182, 310 (2009).

${ }^{27}$ M. Barnes, I. G. Abel, W. Dorland, D. R. Ernst, G. W. Hammett, P. Ricci, B. N. Rogers, A. A. Schekochihin, and T. Tatsuno, Phys. Plasmas 16, 072107 (2009).
${ }^{28}$ P. Helander and D. J. Sigmar, Collisional Transport in Magnetized Plasmas (Cambridge University Press, Cambridge, 2002).

${ }^{29}$ P. J. Catto and D. R. Ernst, Plasma Phys. Controlled Fusion 51, 062001 (2009).

${ }^{30}$ D. R. Ernst, N. Basse, W. Dorland, C. L. Fiore, L. Lin, A. Long, M. Porkolab, K. Zeller, and K. Zhurovich, "Identification of TEM turbulence through direct comparison of nonlinear gyrokinetic simulations with phase contrast imaging density fluctuation measurements," Proceedings of the 21st International Atomic Energy Agency Fusion Energy Conference, Chengdu, China, 2006. 